TUM-HEP-202/94

hep-th/9410205

\title{
S-DUAL GAUGINO CONDENSATION AND SUPERSYMMETRY BREAKING
}

\author{
Z. Lalak ${ }^{\dagger}$, A. Niemeyer ${ }^{\dagger}$ and H. P. Nilles ${ }^{\dagger, \ddagger}$ \\ $\dagger$ Physik Department \\ Technische Universität München \\ D-85748 Garching, Germany \\ ‡ Max Planck Institut für Physik \\ Heisenberg Institut \\ D-80805 Munich, Germany
}

\begin{abstract}
The principle of S-duality is used to incorporate gaugino condensates into effective supergravity (superstring) Lagrangians. We discuss two implementations of S-duality which differ in the way the coupling constant is transformed. Both solve the problem of the runaway dilaton and lead to satisfactory supersymmetry breaking in models with a single gaugino condensate. The breakdown of supersymmetry is intimately related to a nontrivial transformation of the condensate under T-duality.
\end{abstract}

TUM-HEP-202/94

September 1994 


\section{Introduction}

Until now no satisfactory solution for realistic SUSY breakdown in superstring inspired supergravity models has been found. The main flaw of the models considered in the literature is the difficulty of fixing the dilaton, the field which sets the value of the gauge coupling at the unification scale, at a physically acceptable value. Solutions proposed so far in the context of gaugino condensation involve several gaugino condensates and an unnatural adjustment of the hidden matter sector [1]. However it seems possible to rectify the problem of the runaway dilaton in a much more fundamental way by invoking a new symmetry of the constituent Lagrangian, the so-called S-duality.

Gaugino condensation in itself is inherently a field theoretical phenomenon and it might be that S-duality gives the proper way of promoting it into the string theoretical framework. S-duality invariant effective purely dilatonic superpotentials have been conjectured in [2] (however with no reference to gaugino condensation) and recently reexamined by the authors of [3]. The general form of the superpotential proposed in [2], which was constructed specifically to fix the vev of the dilaton, does not give a free theory in the weak coupling limit. The authors of Ref. [3] note that one can easily modify any effective superpotential in such a way, that it vanishes asymptotically as $\operatorname{Re} S \rightarrow \infty$ in any direction. Their one-condensate model shows a realistic minimum for the dilaton but, unfortunately, SUSY is unbroken at this minimum.

As gaugino condensation seems to be the most likely source of the nonperturbative superpotential for the dilaton [4], it is important to understand its role within S-dual string effective actions. Therefore we study the dynamics of supersymmetry breaking in a system containing both condensate and dilaton degrees of freedom as well as the generic modulus $T$ (the breathing mode common to all compactifications).

To construct the S-duality invariant form of the superpotential we note that it has to contain a term originating from the gauge kinetic term $\left(f \mathcal{W}_{\alpha} \mathcal{W}^{\alpha}\right)_{F}(f$ is the gauge kinetic function $\left(\operatorname{Re} f=1 / g^{2}\right)$ and the chiral superfield $\mathcal{W}_{\alpha} \mathcal{W}^{\alpha}$ contains the gaugino bilinear as its lowest component) in the constituent Lagrangian. In order for this term to be invariant under S-duality one has to assume a specific transformation law for the condensate superfield. 
In its simplest realization, S-duality is an $S L(2, Z)$ symmetry generated by $S \rightarrow 1 / S, S \rightarrow$ $S+i$. We shall discuss two physical realizations of S-duality which differ in the way the coupling constant transforms under the action of the first generator:

Type-I S-duality: this is described by $S \rightarrow 1 / S$ and $f \rightarrow f$

(or equivalently $\left.g_{n p}^{2} \rightarrow g_{n p}^{2}\right)$ 円.

Type-II S-duality: defined by the condition $f \rightarrow 1 / f$ (or equivalently $g_{n p}^{2} \rightarrow 1 / g_{n p}^{2}$ ).

This second possibility demands the introduction of an additional field beyond the gaugino condensate which could be called 'magnetic condensate' $H$. Under the type-II transformation $g_{n p}^{2} \rightarrow 1 / g_{n p}^{2} H$ and $Y$ would be exchanged. This is an interesting possibility, however, it is not obvious whether an $N=1$ supersymmetric low-energy field theoretical description is reliable in this case.

\section{Type-I S-duality}

The simplest choice is to consider a tree-level duality invariant condensate. This forces one to assume that the full nonperturbative gauge kinetic function $f$ is also invariant under S-duality.

The correspondence to the weak coupling scattering amplitude calculations demands that $f \rightarrow S$ as $S \rightarrow \infty$. A form of $f$ which fulfills this requirement is

$$
f=\frac{1}{2 \pi} \ln (j(S)-744)
$$

where $j$ is the generator of modular functions of weight 0 . This means that the effective nonperturbative coupling constant is different from $1 / \sqrt{\operatorname{Re} S}$ which has some consequences we will discuss later. Fig 1 shows the behavior of the nonperturbative coupling constant $g_{n p}^{2}$ as a function of $S$.

\footnotetext{
${ }^{1} g_{n p}$ denotes the nonperturbative coupling constant (tree-level plus nonperturbative contributions).
} 


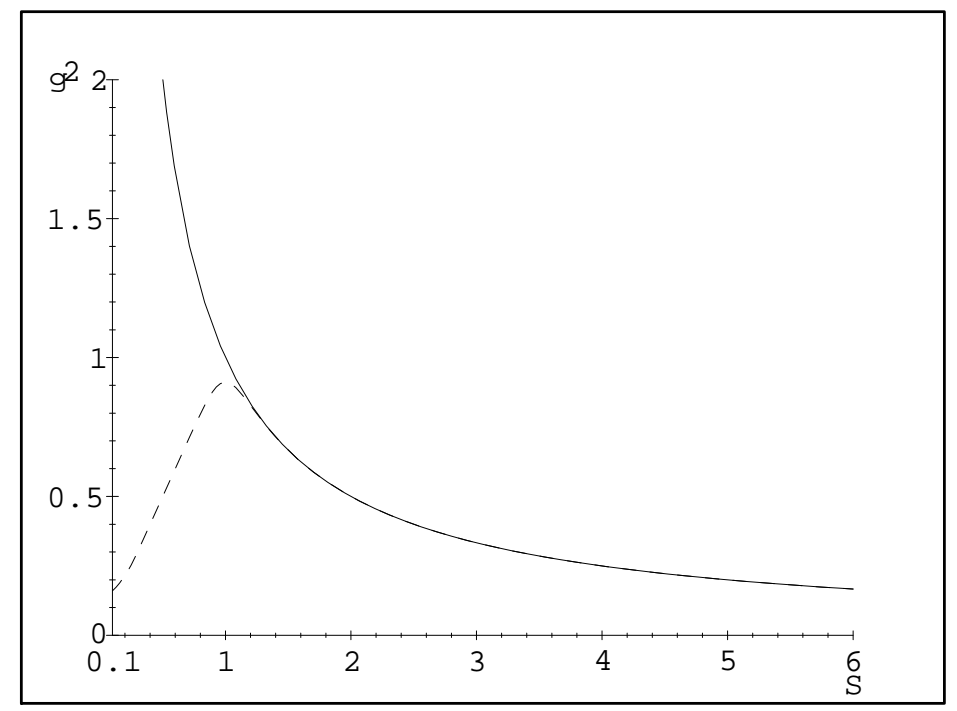

Fig. 1 - Nonperturbative coupling constant $g_{n p}^{2}$ as the function of $S$ in type-I models (dashed) vs $g^{2}$ given by $f=S$

S-modular invariance demands that the superpotential transforms as a modular form of weight -1 (assuming the standard Kähler term $-\ln (S+\bar{S})$ ). The simplest generalization of the Veneziano-Yankielowicz superpotential [5] is then

$$
W=\frac{Y^{3}}{\eta^{2}(S)}\left(\frac{1}{2 \pi} \ln (j(S)-744)+3 b \ln \left(Y \eta^{2}(T) / \mu\right)+c\right),
$$

with $Y^{3}=\mathcal{W}_{\alpha} \mathcal{W}^{\alpha}$. For simplicity we assume $Y$ to transform under $T$-duality like a generic matter field of modular weight -1 . The Kähler function is then of the simple form

$$
K=-\ln (S+\bar{S})-3 \ln (T+\bar{T}-Y \bar{Y})
$$

where $b$ is the usual group theoretical constant, which we take to be 0.1 , and $\mu$ is the order of magnitude where we expect the condensate $Y$ to form. We take it to be $\mu=10^{-5}$ in Planckian units. We will denote the actual vev of the condensate with $\mu^{\prime}$. We adjust the constant $c$ in the way that for $S, T=1$ the gaugino condensate assumes an expectation value of exactly $Y=\mu$. Note that $c$ can be reabsorbed into the scale $\mu$.

This model gives rise to a scalar potential which becomes infinitely large as $S \rightarrow \infty$ in a generic direction (and $S \rightarrow 0$, because of S-duality). However, it is straightforward to verify 
that $V \rightarrow 0$ if the fields go to infinity along the direction given by the condition $W_{Y}=0$. Fig. 2 shows the scalar potential along this quasi-flat direction (because $\left|W_{Y}\right|^{2}$ is the leading term in the scalar potential for small $Y$ ) for fixed value of $T$. Running $S$ to infinity along this valley causes $Y$ to vanish asymptotically. If we write the effective superpotential on the hyper-surface $W_{Y}=0$, which means integrating out the condensate in the usual way [6], we get

$$
W=\frac{\text { const }}{(j(S)-744)^{1 / 2 \pi b} \eta^{2}(S) \eta^{6}(T)}
$$

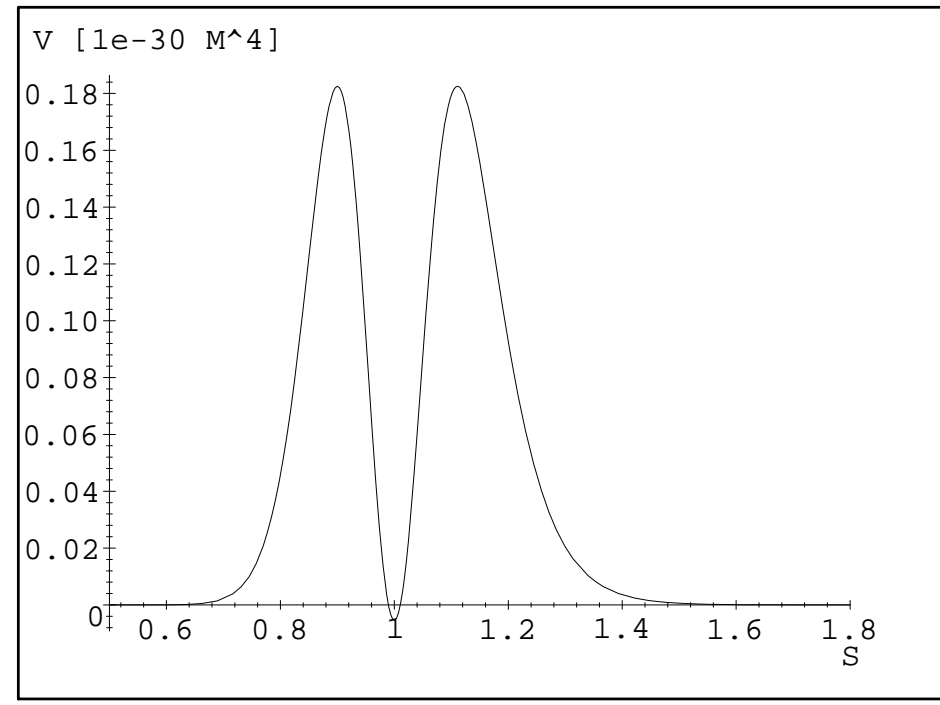

Fig. 2-Scalar potential along the quasi-flat direction in type-I models for a fixed value of T. The potential falls off in the weak coupling regime

This resembles the form conjectured in Ref. [3] except for the different functional behavior in $j$ and most importantly the $T$-dependent term (in [3] no modulus field is included) in the denominator. It differs from the type of superpotential proposed in Ref. [2], where a regular superpotential is required, whereas (in the context of gaugino condensation) we see ourselves forced to admit singularities at finite values of $S$ (though not on the real axis).

It is interesting to note that the weak-coupling limit of our model is different from that of Ref. [2] and the one of Ref. [3]. In Ref. [2] (where gaugino condensates are not considered) $V \rightarrow \infty$ for $S \rightarrow \infty$ regardless of the direction one follows in field space. The authors of Ref. 
[3] on the other hand argue that for gaugino condensates one should have vanishing scalar potential in the weak-coupling limit and consider potentials where $V \rightarrow 0$ for $S \rightarrow \infty$ because of inclusion of a factor of $1 / j$. This prescription gives uniformly vanishing scalar potential in this limit regardless of the actual field content of the model. Our solution gives $V \rightarrow \infty$ for a generic direction but $V \rightarrow 0$ along the quasi-flat direction $W_{Y}=0$, which corresponds to integrating out the condensate. We note at this point, that there is no physical principle which enforces such a strong uniform suppression of the potential as implied by the overall inverse power of $j$. Thus it might be that the requirement implied in Ref. [3] is too strong. The physical condition which has to be imposed on the model is that the expectation value of the gaugino condensate vanishes in the weak-coupling limit, as it happens in our type-I model (as will be seen to happen also in our type-II model).

But because we cannot avoid singularities one could also argue that the $1 / \eta^{2}(S)$ prefactor needed to give $W$ the correct modular weight, could be modified to be of the form $1 /\left(\eta^{2}(S) P\left(j(S)^{1 / 3}\right)\right)$ with $P$ being a polynomial. Including such a factor, e.g.

$$
W=\frac{Y^{3}}{\eta^{2}(S) j^{1 / 3}(S)}\left(\frac{1}{2 \pi} \ln (j(S)-744)+3 b \ln \left(Y \eta^{2}(T) / \mu\right)+c\right) .
$$

makes the potential vanish in the weak coupling limit regardless of the direction one follows in field space.

Minimization of the scalar potentials belonging to these two models reveals that in both cases supersymmetry is broken (in contrast to [3], where the modulus $T$ is not included) $]$. The minimum, which is a global minimum, lies at $S=1, T \simeq 1.23, Y=\mu^{\prime} \simeq \mu$. The supersymmetry breaking scale is determined by the auxiliary field of the modulus $T:\left\langle F_{T}\right\rangle \simeq$ $\mu^{\prime 3} / M$, whereas $\left\langle F_{S}\right\rangle=0$ and $\left\langle F_{Y}\right\rangle \simeq \mu^{\prime 4} / M^{2}$. The cosmological constant is negative and of the order $V_{0} \simeq-\mu^{\prime 6} / M^{2}$. The value of the nonperturbative gauge coupling constant at this minimum is $g^{2}=0.91$.

\footnotetext{
${ }^{2}$ In Ref. [3] supersymmetry breaking is achieved using multiple gaugino condensates.
} 


\section{Type-II S-duality}

The type-II implementation of S-duality takes into account the fact that the gaugino sector of the theory might not close under the S-duality transformation. To write down a model which is invariant one has to include an additional sector, the 'magnetic condensate', which is supposed to represent the dual phase of the theory [7].

The simplest toy model which illustrates the idea of type-II S-duality is given by

$$
\begin{gathered}
K=-\ln (S+\bar{S})-3 \ln (T+\bar{T}-Y \bar{Y}-H \bar{H}), \\
W=\frac{1}{\eta^{2}(S)}\left(Y^{3} S+H^{3} / S+3 b Y^{3} \ln \frac{Y \eta^{2}(T)}{\mu}+3 b H^{3} \ln \frac{H \eta^{2}(T)}{\mu}+Y^{3} H^{3} / \mu^{3}\right) .
\end{gathered}
$$

This model does not exhibit a full $S L(2, Z)$-symmetry, but only the strong-weak-coupling duality

$$
f=S \rightarrow 1 / S, \quad Y \leftrightarrow H
$$

In principle one could with some effort promote this symmetry to a full $S L(2, Z)$, but for illustration of our statements we choose this simple model, especially because both real and imaginary parts of $S$ already become fixed even with this smaller symmetry.

The scalar potential possesses a (although rather hard to find) minimum close to $S=$ $1, T=0.560, H=Y=\mu^{\prime}=3.6410^{-2} \mu$. It turns out that at the minimum supersymmetry is broken, with the magnitude of SUSY breaking again determined by $\left\langle F_{T}>\simeq \mu^{\prime 3} / M\right.$, where $\mu^{\prime}$ is the dynamically determined value of the condensate at the minimum (in the previous type-I examples we adjusted (using $c$ ) $\mu^{\prime}$ to be of the phenomenologically reasonable value $10^{-5}$ ). The cosmological constant is again negative and of the order $\simeq-\mu^{\prime 6}$.

Of course it would be interesting to obtain an effective superpotential containing only dilaton and modulus as we did in our type-I model. One possibility would be to integrate out $Y$ and $H$-fields using the standard conditions $W_{Y}=W_{H}=0$. These conditions are the equations of motion for $Y$ and $H$ in global SUSY and also mean that supersymmetry is unbroken in the naive global limit. Integrating out $Y$ and $H$ is valid because they are 
much heavier than $S$ and $T$, which can be read off the Hessian at the minimum and because their contribution to SUSY breaking is negligible. The procedure of integrating out using the relations $W_{Y}=W_{H}=0$ is valid only if $Y$ and $H$ stay small with respect to the Planck scale, because only then the terms $\left|W_{Y}\right|^{2}$ and $\left|W_{H}\right|^{2}$ are the dominant contributions to the scalar potential. These are given by

$$
\begin{aligned}
& W_{Y}=\frac{3 Y^{2}}{\eta^{2}(S)}\left(S+3 b \ln \frac{Y \eta^{2}(T)}{\mu}+b+\frac{H^{3} \eta^{6}(T)}{\mu^{3}}\right) \\
& W_{H}=\frac{3 H^{2}}{\eta^{2}(S)}\left(\frac{1}{S}+3 b \ln \frac{H \eta^{2}(T)}{\mu}+b+\frac{Y^{3} \eta^{6}(T)}{\mu^{3}}\right)
\end{aligned}
$$

One cannot solve both conditions simultaneously in an analytical way. Since we are interested in the nature of the weak-coupling regime in this model, we solve the above equations asymptotically for large $S$. It is easy to see that in order to preserve $W_{Y}=0, Y$ has to go exponentially to 0 as $S \rightarrow \infty$. Inserting this result into the second equation one obtains two solutions: $H \rightarrow \exp (-\alpha S)$ with a sufficiently large $\alpha$ (say 1 ), or $H$ staying finite and always exactly cancelling the bracket in (10), going ultimately to $\exp (-1 / 3) \mu / \eta^{2}(T)$. In order to use these relations we had to assume $Y, H$ to be small, which we consistently get in both solutions for large $S$. After expressing the superpotential in terms of $W_{Y}, W_{H}$

$$
W=Y W_{Y}+H W_{H}-\frac{1}{\eta^{2}(S)}\left(3 b(Y+H)+Y^{3} H^{3} \eta^{6}(T) / \mu^{3}\right)
$$

one can see that for $S \rightarrow \infty$ the superpotential in the first case goes to zero exponentially, but blows up in the second case $\left(1 / \eta^{2}(S)\right.$ behaves asymptotically like $\left.\exp (\pi S / 12)\right)$. This implies in turn that the scalar potential $V$ for large $S$ becomes 0 in the first or infinitely large in the second case, in contrast to our type-I model. Taking the usual conditions for eliminating condensates one can therefore arrive at different effective superpotentials.

However, this could be a very model dependent property. If one wishes to have vanishing scalar potential in the weak coupling limit, one could always include e.g. an $1 / j(S)$ factor in the superpotential. In addition we would like to point out that we only considered the direction along which SUSY is not broken by the auxiliary fields of the condensates. 


\section{Discussion and Conclusions}

Our analysis shows that it is possible to have realistic SUSY breaking in type-I models with a single gaugino condensate and without matter fields by invoking S-duality. However, Sduality does not seem to solve the problem of the cosmological constant, which is negative in both types of models. It can be shown that even small non-S-dual perturbations of the superpotential preserve the nice features of the above models [8]. Maybe they could be used to cancel the cosmological constant.

Considering more general modular invariant functions instead of $j^{-1 / 3}$ in (5) might lead to a change of the magnitude of the supersymmetry breaking scale. If the modulus $T$ is absent from the theory, the minima are still well defined, but SUSY stays unbroken.

Note that the analysis for type-I models is based on the assumption that the condensate does not transform under S-duality. In principle it would be possible to assume a nontrivial transformation behavior for the $Y$-field. However, one is forced to constrain oneself to $Y$ and $f$ transforming as modular forms of opposite weight, because with the minimal choice of variables in the superpotential $(S, Y)$ it is impossible to cancel an arbitrary transformation law in the term $f Y^{3}$. In order to have $T$ transforming trivially under S-duality (as it should at tree-level), one has to include appropriate $\eta$-factors in the logarithm in the Kähler function, if $Y$ does transform as some modular function of $S$. However, one can then see that it is easy to redefine $Y$ and $f$ by shifting some power of $\eta$-functions from one to the other, so that one gets back to the form we proposed above.

For our type-II model several desirable properties are the same as for the type-I models: SUSY is again broken at a reasonable minimum, one observes no supersymmetry breaking if one does not include the modulus $T$ and the scale of SUSY breakdown is similar in both types of models as is the cosmological constant. The weak-coupling limit however, is different. Type-I models generically lead to vanishing scalar potential in the limit $g^{2} \rightarrow 0$ after eliminating the condensate through its equations of motion. This is not the case for our type-II model, as the integration conditions have different solutions with different asymptotic behavior, but there seems to be no argument why the potential has to vanish in the $g^{2} \rightarrow 0$ 
limit, because asymptotically the gaugino condensate does vanish, as required. The magnetic condensate $H$ may not vanish together with the gaugino condensate and therefore there is no a priori quasi-flat direction in this model in the weak-coupling regime. This also shows that the usual conditions to eliminate condensates might have to be revised.

We have not considered stringy one-loop corrections here. These usually introduce a mixing of $S$ and $T$ in the Kähler function, so that $T$ cannot transform trivially under Sduality any longer. One could imagine that this problem can be solved by introducing two new orthogonal fields $S^{\prime}, T^{\prime}$ which do not mix under their respective duality transformations. This would be in analogy to the usual redefinition of the dilaton in $T$-modular invariant one-loop-gaugino condensation models.

We have demonstrated that the idea of S-duality applied to gaugino condensation induced stringy superpotentials naturally leads to potentials with well-defined supersymmetry breaking minima. We have proposed two different ways of incorporating S-duality into the low-energy effective Lagrangian, which differ in the way the coupling constant is transformed, but both lead to physically satisfying solutions while employing only a single gaugino condensate. We feel that this solution to the long-standing problem of runaway dilaton vacua is more attractive than the traditional methods relying on multiple condensates. In addition we have shed some light on the necessity of having a vanishing scalar potential in the weak-coupling limit.

\section{Acknowledgments}

This work was supported by the Deutsche Forschungsgemeinschaft and EC grants SC1-CT920789 and SC1-CT91-0729. Z.L. has been supported by the A. von Humboldt Fellowship. A. N. has been supported by a $\mathrm{PhD}$ scholarship from the Technical University of Munich. We would like to thank Andre Lukas and Peter Mayr for useful discussions. 


\section{References}

[1] N.V. Krasnikov, Phys.Lett. 193B (1987) 37; J.A. Casas, Z. Lalak, C. Muñoz, G.G. Ross, Nucl.Phys. B347 (1990) 243; for an extended list of references see: B. de Carlos, J.A. Casas, C. Muñoz, Nucl.Phys. B399 (1993) 623

[2] A. Font, L. Ibáñez, D. Lüst, F. Quevedo, Phys.Lett. 249B (1990) 35; J. Schwarz, A. Sen, Nucl.Phys. B411 (1994) 35; for an extended list of references see: A. Sen, Int.J.Mod.Phys A9 (1994) 3707

[3] J.H. Horne, G. Moore, "Chaotic Coupling Constants", hep-th/9403058

[4] H.P. Nilles, Phys.Rev.Lett. 115B (1982) 193; S. Ferrara, L. Girardello, H.P. Nilles, Phys.Lett. 125B (1983) 457; J.P. Derendinger, L.E. Ibáñez, H.P. Nilles, Phys.Lett. 155B (1985) 467; M. Dine, R. Rohm, N. Seiberg, E. Witten, Phys.Lett. 156B (1985) 55; T.R. Taylor, Phys.Lett. 164B (1985) 43

[5] G. Veneziano, S. Yankielowicz, Phys.Lett. 113B (1982) 231

[6] D. Lüst, T.R. Taylor, Phys.Lett. 253B (1991) 335

[7] C. Montonen, D. Olive, Phys.Lett. 72B (1977) 117; C. Vafa, E. Witten, "A strong coupling test of S-duality", hep-th/9408074

[8] Z. Lalak, A. Niemeyer, H.P. Nilles, in preparation 